\title{
Using ground relays for low-latency wide-area routing in megaconstellations
}

\author{
Mark Handley, University College London
}

\begin{abstract}
Large low earth orbit satellite constellations have the potential to provide global high-capacity low-latency Internet access, but early deployments lack dedicated inter-satellite links (ISLs). We investigate the use of ground-based relays as a substitute for ISLs to provide low-latency wide area networking. We examine how to route such huge networks, potentially using user-terminals as relays, and devise an enhanced routing algorithm to cope. We show that even without ISLs, such networks can still beat optical fiber networks for latency.
\end{abstract}

\section{INTRODUCTION}

Several companies have filed plans to build large Low Earth Orbit (LEO) constellations to provide high-bandwidth lowlatency Internet service. SpaceX and OneWeb have launched their first satellites $[11,6]$, with SpaceX launching 60 satellites into the first orbital plane of their Starlink network. The satellites are not the final version; they have phased-array antennas allowing simultaneous communication with multiple ground stations, but lack laser-based inter-satellite links (ISLs) that others have simulated [4, 7]. This has lead many to question whether these first constellations have any role to play in providing low-latency wide area communications.

Laser ISLs are planned for second generation satellites [8], but current state-of-the-art free-space laser links [13] support lower data rates than Starlink's RF link capacity, potentially leading to ISLs still being a bottleneck. An alternative to ISLs is to relay between satellites via a ground station. In this paper we examine the potential role for ground relays in Starlink. We examine three scenarios:

- Early and mid phase one deployment, without ISLs.

- Full phase one (1584 satellites), without ISLs.

- Full phase one, using both ISLs and ground relays.

We investigate what performance is possible: we look especially at the case when dense networks of user terminals can be used as relays, but also examine trans-oceanic paths with few relays. We suggest routing algorithm enhancements that make such networks feasible to consider.

\section{STARLINK AND GROUND RELAYS}

According to public filings [9] with the US Federal Communications Commission (FCC), SpaceX's original plans for the first phase of Starlink involved 1600 satellites, consisting

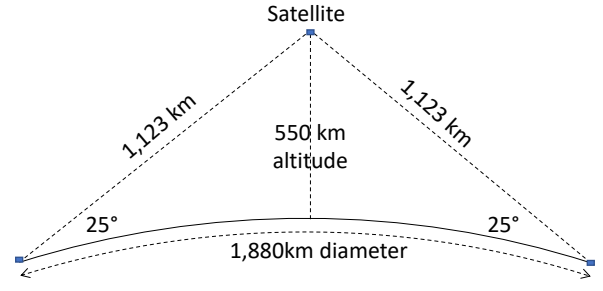

Figure 1: Limits of coverage

of 32 orbital planes each containing 50 equally spaced satellites, with these orbital planes inclined at $53^{\circ}$ from the equator and with the satellites orbiting at an altitude of $1100 \mathrm{~km}$. In prior work [4] we evaluated this suitability of this constellation with regards to its ability to provide low-latency widearea communications. This original design was well suited to ISLs, with distances between satellites in the same plane being similar to those between neighboring planes, permitting a relatively "square" mesh of laser links to be formed. We concluded that it should indeed be possible to use such a constellation to achieve lower latencies than optical fiber, so long as the endpoints were more than roughly a thousand kilometers apart.

In November 2018 SpaceX filed revised plans with the FCC, lowering the altitude of the satellites to $550 \mathrm{~km}$, using fewer orbital planes (24 rather than 32) but increasing the number of satellites per plane from 50 to 66, thus keeping the total number of satellites roughly the same. The orbital inclination of $53^{\circ}$ remained unchanged [10].

The revised constellation is much less symmetric: neighboring satellites in the same orbital plane are much closer to each other than to the corresponding satellite in the next orbital plane. This first phase will no longer use laser ISLs: the technology is in its infancy[13], and it is likely that the laser-links were simply not ready in time.

In SpaceX's revised November 2018 FCC filings, the angle at which a satellite is reachable was reduced from $40^{\circ}$ above the horizon to $25^{\circ}$, corresponding to an increase in the angle the satellite must be able to steer a spot-beam from the vertical to reach a groundstation. Such high slant angles increase the coverage radius of each satellite, but at a cost of lower signal strengths and decreasing spatial reuse of frequencies due to potential interference from other satellites in a similar direction. A key benefit though is that a low orbit combined with high slant angle regains some of the low latency properties that previously required laser ISLs[4]. Fig. 1 shows the coverage of a satellite in a $550 \mathrm{~km}$ orbit, reachable at $25^{\circ}$ above the horizon. In the limit, two ground- 


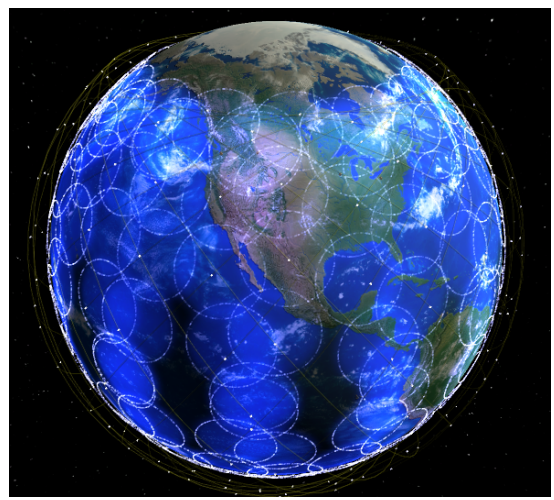

Figure 2: 11 satellites $\times \mathbf{2 4}$ planes

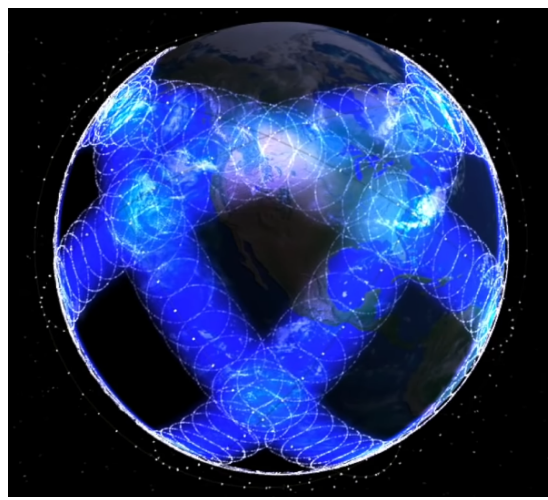

Figure 3: 66 satellites $\times 6$ planes

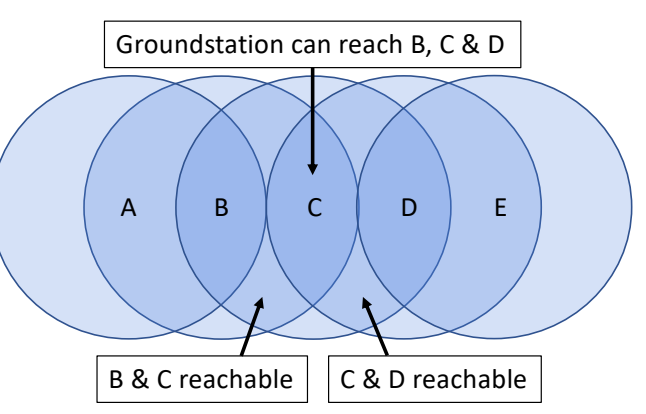

Figure 4: Coverage overlap with 66 satellites per plane stations $1880 \mathrm{~km}$ apart can communicate via a single satellite, with a path length of $2,246 \mathrm{~km}$. The speed of light latency would be $7.5 \mathrm{~ms}$ via the satellite, whereas through optical fiber with a refractive index of 1.468[2] laid in a straight path on the Earth's surface, the latency would be $9.2 \mathrm{~ms}$. Although this is the limiting case, it does open up the possibility of achieving low latency long distance communication, even without ISLs, so long as there are always ground relay stations in suitable locations. How feasible is this in practice? Is it possible in the early stages of constellation deployment, or does it require high satellite density? To answer this question, we simulated the SpaceX Starlink network in a range of possible configurations.

\subsection{Partial Deployment Performance}

If continuous coverage of the maximum ground area were the goal, partial deployment would spread satellites out over multiple orbital planes, with fewer satellites per plane.

Fig. 2 shows the $550 \mathrm{~km}$ orbits from the SpaceX FCC filing, partially populated with 264 satellites comprising 11 satellites in each of 24 orbital planes. This constellation provides continuous coverage over the continental US. However, most areas can only see one satellite at a time, rendering groundstations there unable to relay traffic.

In contrast, Fig. 3 shows six orbital planes with 66 satellites per plane. The Earth rotates under these planes, but there is a band around $50^{\circ} \mathrm{N}$ and one at $50^{\circ} \mathrm{S}$ that have continuous coverage. In North America, the band covers the US from New York City northwards and much of Canada. Most of Europe is also covered, with the exception of southern Spain, southern Italy, Greece, and northern Scandinavia.

At this altitude and reachable elevation angle, 66 satellites per plane hits a sweet spot where the diameter of a satellite's zone of coverage overlaps $2 / 3$ with that of the next satellite in the plane. As shown in Fig. 4, this means that most places that have coverage can see at least two satellites at a time.

We simulated possible early deployment scenarios for the Starlink network without ISLs to investigate long-distance service latency, and to investigate the impact of relay place- ment. We simulated six orbital planes of 66 satellites, which is the minimum number of planes to provide continuous service to any locations, 12 orbital planes, which can provide continuous service to the continental US, Japan, and Europe below $60^{\circ} \mathrm{N}$, and the full 24 orbital planes which can provide global coverage from $60^{\circ} \mathrm{N}$ to $60^{\circ} \mathrm{S}$. To determine the best relay placement, we configured a grid of potential relays and used Dijkstra's algorithm to determine the lowest latency path using any of the relays. 1,250 potential relays are simulated, spaced at $1^{\circ}$ intervals from $75^{\circ} \mathrm{W}$ to $122^{\circ} \mathrm{W}$ and from $30^{\circ} \mathrm{N}$ to $55^{\circ} \mathrm{N}$, covering most of the US and Southern Canada in a roughly $95 \mathrm{~km}$ grid. Fig. 5 shows the path from New York to Seattle. Both these cities lie in the band of continuous coverage for six orbital planes, though New York is only just in this band. As the Earth rotates under the orbital planes and as the satellites traverse their orbits, the locations of the optimal relays change. The locations shown in yellow are the ones used as relays during one four-hour period, after which the routing paths approximately repeat.

Fig. 6A shows how the RTT varies with time when routing between these cities using the near-optimal relay locations. For comparison, the best case RTT using optical fiber stretched tight along the great circle route ${ }^{1}$ is $38 \mathrm{~ms}$ and the current Internet RTT is $76 \mathrm{~ms}[12]$. Essentially there are two overlayed cycles happening here. The long timescale cycle is caused by the Earth rotating under the orbital planes. With six planes, this has a period of four hours, and is proportionally shorter with 12 and 24 planes. The short timescale cycle is caused by satellites passing overhead. The 66 satellites in a plane complete an orbit in 5739 seconds, so the period of this variation is $5739 / 66=87$ seconds. The large steps in RTT are caused when the path switches from one plane to another as a satellite on the best path moves out of range, or a satellite moves into range allowing a better path.

Using the optimal locations from Fig. 5 as a heat map and adding extra relays as needed to ensure connectivity, we configured ten relays across the US and Canada, each located in a population center. Fig. 6B shows the RTT using just these

${ }^{1}$ This is not possible in practice 

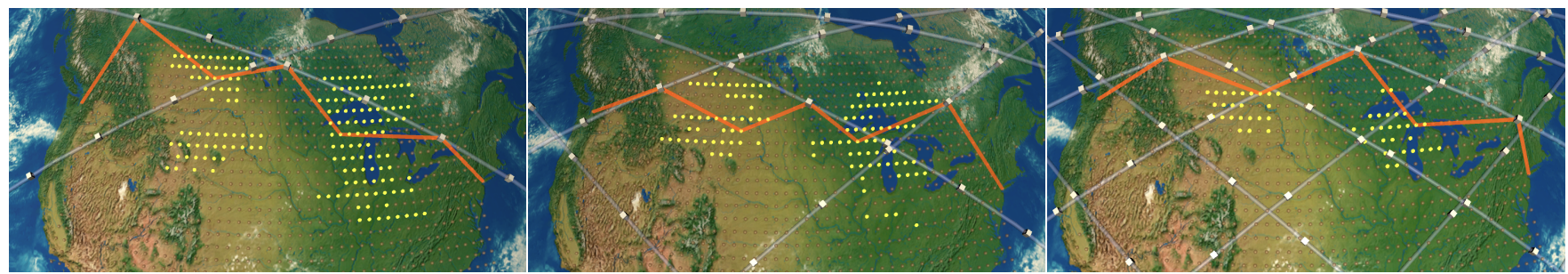

Figure 5: Spread of best relay locations for NY-Seattle. 6 planes (left), 12 planes (middle) and 24 planes (right)
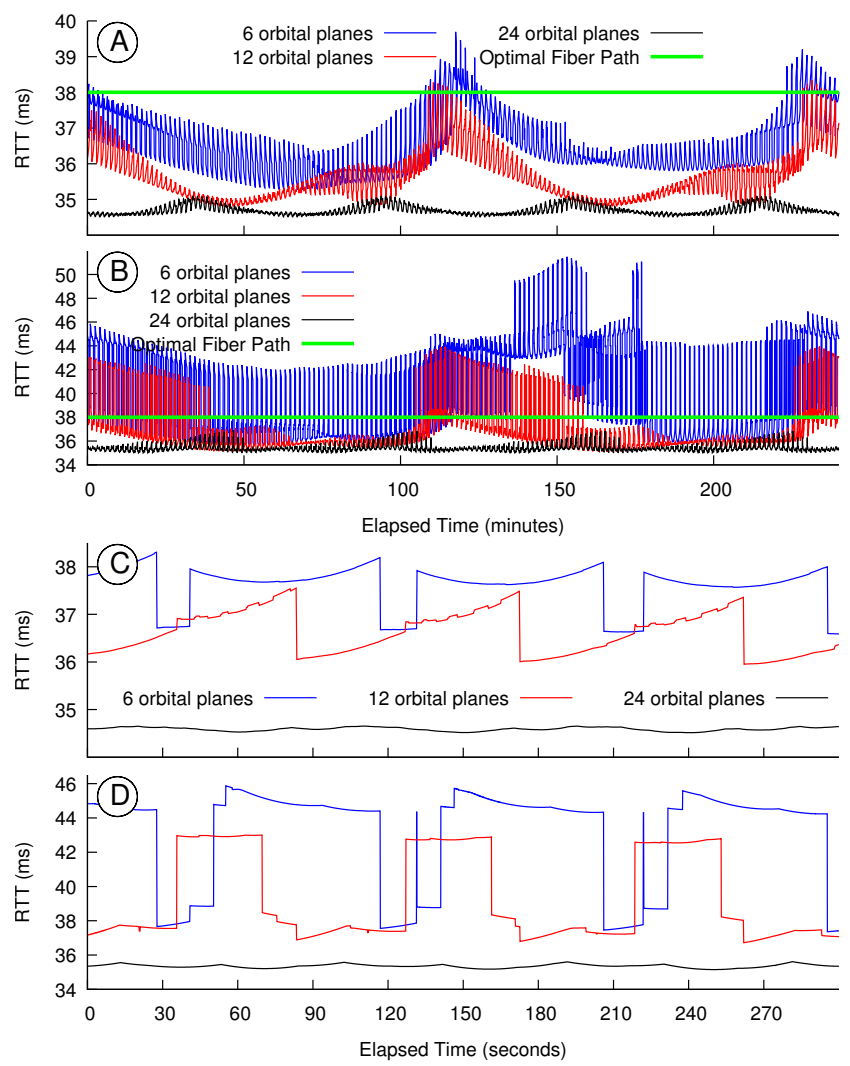

Figure 6: NY-Seattle RTT, (A) 4 hours using 1250 relays with $1^{\circ}$ spacing, (B) 4 hours using 10 relays, (C) 5 minutes using 1250 relays with $1^{\circ}$ spacing, (D) 5 minutes using 10 relays

ten relays. Latency is still lower than current Internet RTTs, but using close to the minimum number of relays does impact both latency and variability of latency. This should not be a surprise, especially in the early 6-plane deployment scenario, as sometimes relays are relatively far from an orbital plane. As additional groundstations are added and made available as relays, the latency progressively decreases from that in Fig. 6B towards that in Fig. 6A.

Zooming in on the first 30 seconds, we can see the short term behaviour shown in Fig. 6C and 6D. The 87-second short-term periodicity is evident as each satellite passes over and is replaced by its successor in the orbital plane. The somewhat bimodal latency is due to sometimes two relays being sufficient, and sometimes three being needed. As the

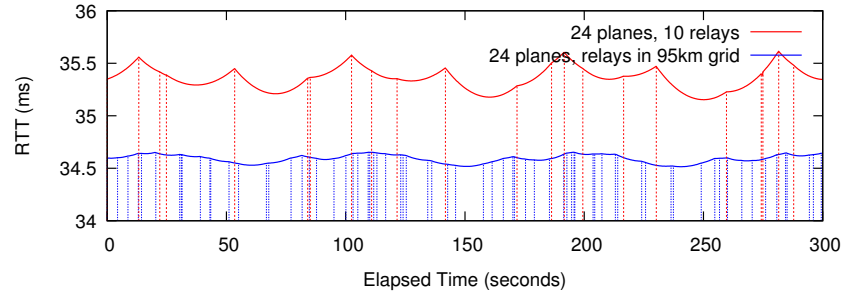

Figure 7: NY-Seattle RTT and route change frequency

constellation is built out and becomes denser, as with 24 planes, two relays are sufficient on this path at all times.

\subsection{Relay Density vs Route Stability}

There are two potential downsides to considering more relays in the routing computation. First, the additional relays inflate the number of links in the network graph. From the routing point of view, a link exists from a groundstation to any satellite that is within range. This can significantly slow down the routing computation. Second, with more relays, the lowest latency route changes more frequently. Fig. 7 shows the same data as the 24-plane curves from Fig. 6C and $\mathrm{D}$, but vertical lines have been added to show when the shortest path route changes. Lower latency goes hand-in-hand with more frequent route changes. If computing routes is expensive, computing them this frequently could be a problem.

How many groundstations will routing need to handle? If relays are provisioned by Starlink, the number might be relatively few, though probably many more than ten. However, SpaceX has filed with the FCC for a spectrum license covering one million US-based user terminals. These terminals will use phased array antennas to generate softwaresteerable spot beams[8], and will be able to communicate with multiple satellites simultaneously - how many is primarily a question of processing power to generate the signals for each antenna element, or to separate simultaneously received signals. User terminals likely will be less capable than SpaceX gateway nodes, as they must be inexpensive. However, it also seems feasible that inactive or lightly loaded user terminals might be used as relays if this only requires them to talk to two satellites at a time. These terminals will run SpaceX software, with all traffic encrypted edge-toedge, so the risks of relaying through them seem manageable.

To examine the effect of density of relays, we configure 


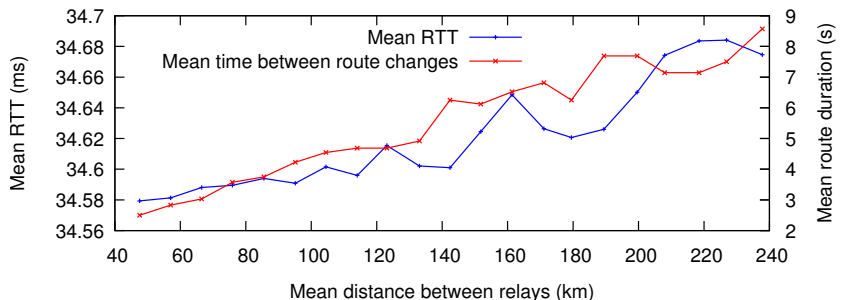

Figure 8: RTT and route change frequency, NY-Seattle, as a function of relay density

the grid of groundstations shown in Fig. 5, but vary the density from one every $2.5^{\circ}$ of longitude and latitude (a mean spacing of $238 \mathrm{~km}$ ) to one every $0.5^{\circ}$ of longitude and latitude (mean spacing of $48 \mathrm{~km}$ ). Fig. 8 shows both the RTT from New York to Seattle (left axis) and the mean time between shortest path route changes (right axis). With more relays, there are more options for shortest path, so the route changes more often. However, above one relay per degree giving an average of $95 \mathrm{~km}$ spacing, there is very little latency benefit, but the time between route changes continues to drop, requiring frequent route calculation to track these changes. This graph also shows an interesting ripple in the RTT - this is caused by aliasing of the fixed spacing between satellites against the much tighter spacing of groundstations, and is an artifact of the excessively regular grid used in this simulation.

\subsection{Trans-oceanic Routes}

While it should be possible to rely on large numbers of ground relays, even in rural parts of North America, the same is not true of the world's oceans. One key advantage of LEO constellations using ISLs is low-latency wide-area intercontinental communications. Without ISLs, is it still possible to provide low latency trans-oceanic communications? One possibility might be to place relays on the fleets of cargo ships that ply the world's oceans, or to use aircraft in flight[1]. While we believe that in time, these may supplement basic connectivity, we also wish to understand how well a network like Starlink can perform using only a few relays on trans-Atlantic and trans-Pacific routes.

Fig. 9 shows the North Atlantic route from New York to London. Relays in eastern Canada and Ireland bring traffic to and from a path across the ocean, but two relay ships located around $53^{\circ} \mathrm{N}, 24^{\circ} \mathrm{W}$ and $51^{\circ} \mathrm{N}, 41^{\circ} \mathrm{W}$ are needed to bridge the gap. These relays are sufficient to provide continuous trans-Atlantic connectivity, even with only 6 orbital planes, because they lie in the $50^{\circ} \mathrm{N}$ dense coverage band. As more orbital planes are added, latency decreases, and with 24 orbital planes latency is always lower than the lower bound for optical fiber along the great circle route. Even with 6 orbital planes, the RTT varies between 55 and $70 \mathrm{~ms}$, which is better than the current 75 ms Internet RTT[12]. A network with ISLs would perform better still, with a mean
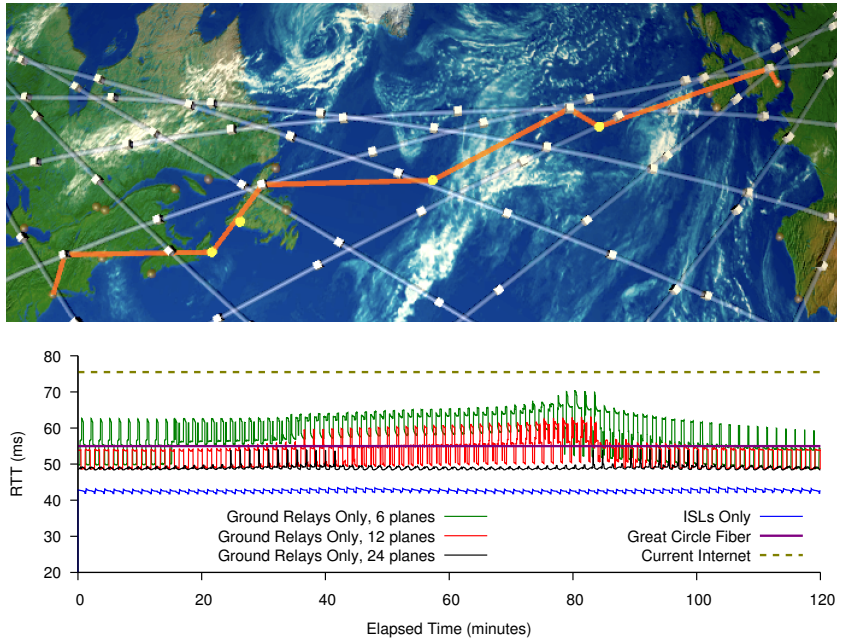

Figure 9: London-New York using two ships
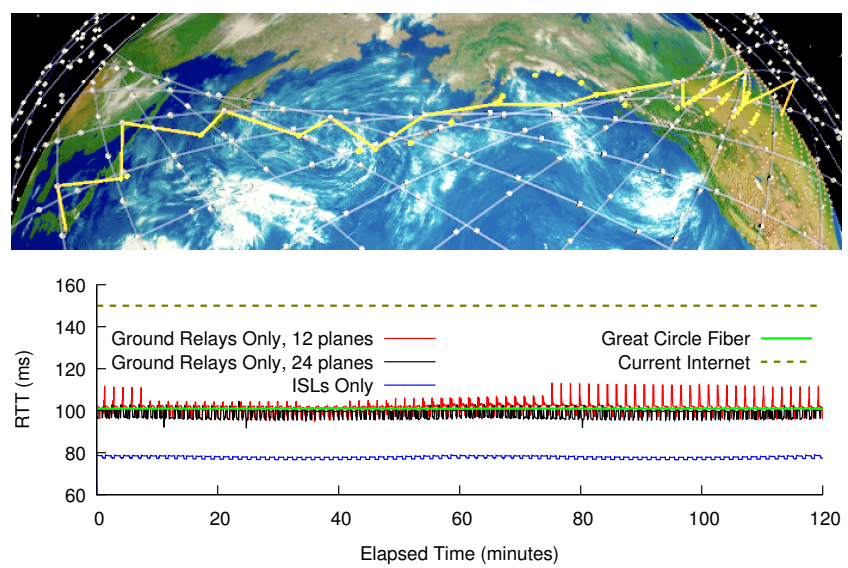

Figure 10: Chicago-Tokyo via the Aleutian Islands

of around $43 \mathrm{~ms}$ compared to $48 \mathrm{~ms}$ using ground relays.

A trans-Pacific ground-relay route is also possible. Fig. 10 shows the Chicago-Tokyo path. Tokyo is not within the continuous coverage zone with 6 orbital planes, but low latency communication is possible with 12 planes or more. The Aleutian Islands are arranged in a very convenient east-west path at just the right latitude to act as relays. Adding relays along the Canada and Alaska coast and Northern Japan almost completes the path. One more relay is needed at the southern tip of the Kamchatka peninsula in Russia's far east. If such a relay is not possible due to political issues, another ship will be required to bridge this gap. Fig. 10 assumes the Russian relay is used, but latency would be similar using a ship. For this path, using 24 planes with relays does not always beat the optimal fiber path, though the satellite path has a lower mean and beats the current Internet path by 50 ms. Again, there would be a clear win for ISLs, but until that technology is ready, ground relays should be a viable solution. 


\section{ROUTING PERFORMANCE}

We use a binary-heap implementation of Dijkstra's algorithm[3] to calculate the lowest latency paths. The routing graph needs to contains an edge from each satellite to the potential relays in range. The limiting factor turns out not to be Dijkstra's algorithm; as the topology changes constantly, most time is spent rebuilding the routing graph from the geographic positions and updating edge latencies. This takes $O(r * s)$ time, where $r$ is the number of relays and $s$ is the number of satellites. For a fixed constellation, the cost of this phase scales linearly with $r$. With 5,000 US-based relays (a mean separation of $48 \mathrm{~km}$ ), building the routing graph and then running Dijkstra's algorithm takes 1.55 seconds on a 2.7Ghz Intel Core i7 CPU. The mean time in this configuration between shortest path route changes is 2.5 seconds, so this is starting to be problematic.

We use a hierarchical update process to reduce the cost of topology updates. First, we divide the Earth's surface into tiles, $k$ degrees longitude by $k$ degrees latitude. Each tile maintains a list of relays from that region. To update the downlinks from a satellite, we check which tiles overlap the circle of coverage of the satellite. All relays within an overlapping tile are added to a list of potential relays. We then iterate over the resultant list, adding each relay as a graph edge to the satellite if the relay is within range.

Building the routing graph in this way is much cheaper than the naive approach, but it is still more expensive than just updating edge latencies in an existing routing graph. We take advantage of this by not rebuilding the topology graph every time we need to calculate routes. Instead, we add a margin of $m \mathrm{~km}$ to the edge of the zone of coverage of the satellite $^{2}$. When we build the routing graph, we add edges for relays within this extended region. Edges to nodes in the margin area are given a link latency of infinity, so Dijkstra will not use them yet.

When recalculating routes, the previous graph is retained, with only edge latencies being updated. Some edges with infinite latency become usable, and some that previously were reachable have moved out of range and become infinite cost. All the rest have their latency updated. Although the margin means more edges need to have their latency cost updated, this is more than offset by not needing to rebuild the graph.

A route graph can be reused in this manner until the satellite has moved more than the size of the margin. For a 100 $\mathrm{km}$ margin and satellites at $550 \mathrm{~km}$ altitude, this takes 13.7 seconds. The route graph must then be rebuilt from the tiles. In our implementation, rebuilding the graph from tiles takes twice as long as updating edge latencies. We do not need to rebuild the route graph for all satellites simultaneously. If routing computations are performed every $500 \mathrm{~ms}$, we only need to rebuild the route graph for less than $4 \%$ of the satellites each time, so the routing cost becomes primarily that of

\footnotetext{
$\overline{{ }^{2} \text { we get best performance with } k}=10^{\circ}$ and $m=100 \mathrm{~km}$
}

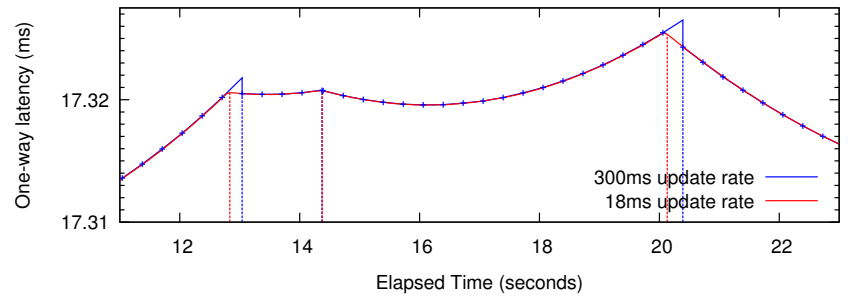

Figure 11: Latency steps due to low update frequency

updating latencies and running Dijkstra.

These optimizations reduce the time taken to route from one source to all destinations in a 1,584 satellite, 5,000 relay network, from 1.55 seconds in the naive implementation to $61 \mathrm{~ms}$. Scaling is almost linear, with each additional relay taking $12.5 \mu \mathrm{s}$. A network with 40,000 relays can be routed in under $500 \mathrm{~ms}$; this is equivalent to relays placed every $60 \mathrm{~km}$ covering the entire land surface of the Earth. As Fig. 8 shows, there is very little latency benefit from using relays closer than this. Of course, user terminals may in some places be much denser, requiring the network controller to elect an appropriately spaced idle subset to use as relays.

Further optimizations are possible. For example, only relays that are close to the optimal location to be used as a relay between a pair of satellites need to be considered by the routing computation. We have, however, not yet found it necessary to perform such optimization.

\subsection{Differential Routing}

Fig. 11 shows the effect of update frequency, looking at 20 seconds from Fig. 7 using relays in a $95 \mathrm{~km}$ grid. The blue curve shows the latency if routing updates are performed every $300 \mathrm{~ms}$, whereas the red curve shows if they are performed every $18 \mathrm{~ms}$. In this case the impact on latency of switching routes too late is not huge $-30 \mu$ s or so - but at $20 \mathrm{~Gb} / \mathrm{s}$ this is still enough to cause reordering by 50 packets.

Such reordering is unnecessary: we can predict accurately when to change paths without needing to run the full routing algorithm with high frequency. Suppose we run the routing algorithm every $500 \mathrm{~ms}$, but run it for the network as it will be in one second's time. This is possible because the orbits are all completely predictable. If we see that between the next two routing updates, the path to a destination has changed, we know that it should change sometime between $\mathrm{t}=500 \mathrm{~ms}$ and $\mathrm{t}=1000 \mathrm{~ms}$, but Dijkstra's algorithm does not tell us precisely when the change should occur.

Link latency changes in a smooth curve, as a satellite closes in on a relay, then moves away. Path latency is the sum of link latency curves, and so is also a smooth function between route changes. We don't need to know the exact function, but wish to calculate when to change routes within a few milliseconds. We can cheaply estimate this by approximat- 

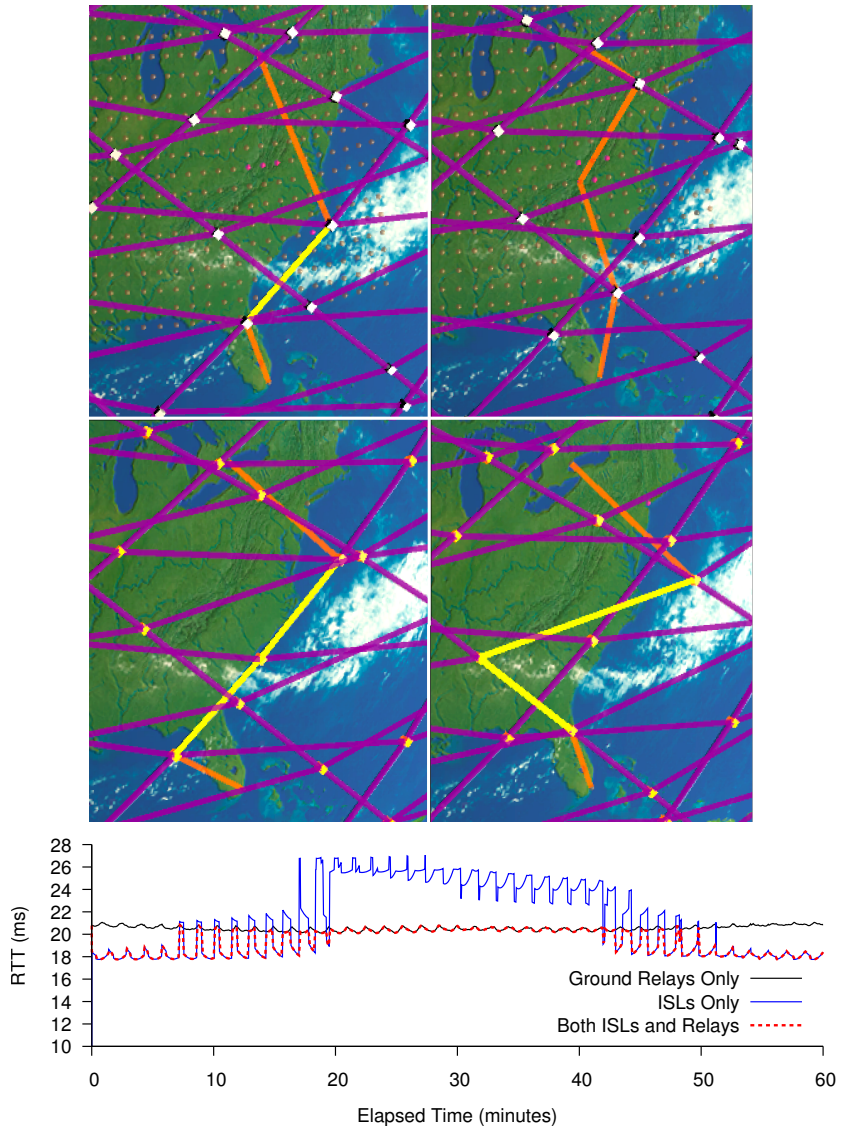

Figure 12: Toronto-Miami

ing the $1^{\text {st }}$ and $2^{\text {nd }}$ derivative of the link latency. Both are easily calculated when updating the link latencies, $l$ :

$$
\dot{l_{n}}=\frac{l_{n}-l_{n-1}}{t_{n}-t_{n-1}}, \quad \ddot{l_{n}}=\frac{\dot{l_{n}}-l_{n-1}}{t_{n}-t_{n-1}}
$$

The path latency, as calculated by Dijkstra, is the sum of the link latencies. Dijkstra's algorithm can also be modified to propagate the sums of first and second derivatives, so that once a route is chosen, it is trivial to estimate how its latency will change over short time intervals, both forwards and backwards in time. If two runs of Dijkstra $500 \mathrm{~ms}$ apart result in two different paths, we can then use the derivatives to determine with high precision when the old path becomes worse than the new path. This allows route changes to take place at almost exactly the optimal moment. Care must also be taken to switch away from a route at the correct time if any extrapolated link latency takes it out of satellite range.

We note that such differential routing is an approximation of the optimal algorithm - if a third route were briefly possible between two route computations, this approximation will not use it. This algorithm will, however, ensure that there is no unnecessary latency step that might cause reordering.

\section{HYBRID NETWORKS}

Using a direct ISL between a pair of satellites will always
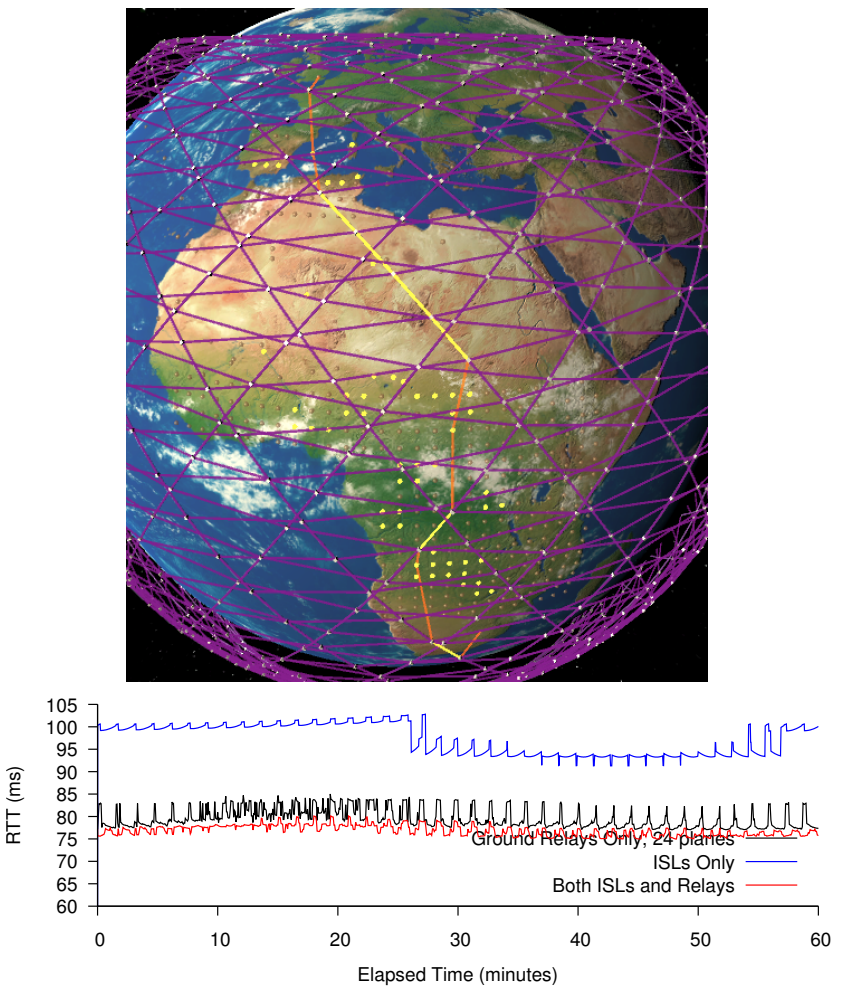

Figure 13: London-Johannesburg

give lower latency than using a relay. In later phases, SpaceX plans to employ laser ISLs between satellites. Is there still a role for ground relays in a LEO constellation employing laser ISLs? Perhaps surprisingly, the answer is yes. From FCC filings[10], we can deduce that SpaceX plans to use four laser ISLs per satellite. If we optimize ISLs for EastWest low latency communications, this results in connecting to the next and previous satellites in the same orbital plane, and to a satellite slightly offset in the neighboring orbital plane. We analysed such topologies in $[4,5]$; they provide good low-latency communication on many paths, and give stable targets for the lasers to track. However, using ISLs in this way can still give sub-optimal latency, as NE-bound satellites only connect to other NE-bound satellites, and SEbound satellites (which are in the same inclination orbital planes, but on the far side of their orbits) only connect to other SE-bound satellites.

Two examples illustrate this sub-optimality. Consider first the path from Toronto to Miami using 24 orbital planes, as shown in Fig. 12. Purple depicts ISLs, yellow is an ISL used on the shortest path, and orange is an RF link from a satellite to a groundstation. Miami is nearly due south of Toronto; the ISLs to neighboring orbital planes are not well oriented for this path, so ISLs along the orbital planes get used.

The graph shows the RTT over an hour, using ISLs, ground relays, or both. The ISL paths are very variable, as ISLs along the orbital plane are never well aligned north-south, so the path is always a zig-zag one. Initially the ISL path 
uses one optical path segment, as in the top left picture, but alternates between NE-bound and SE-bound satellite pairs. As the Earth rotates under the orbital planes, they become less well aligned with the path, and we start to see longer ISL paths with two or sometimes three optical segments being used, as in the lower pictures. Although the path using ground relays (top right picture) performs a vertical zig-zag between satellites and relays, in the horizontal direction it is quite direct. As a result, in cases like this it is better to consider both ISLs and ground relays when computing lowlatency routes, as shown in the red curve in Fig. 12.

Another sort of route where relays assist ISLs is shown in Fig. 13, depicting a snapshot of the route from London to Johannesburg which also runs mostly north-south. In this case, we cannot assume uniform relay coverage across the Sahara, so we chose 25 groundstation locations in population centers. These are very remote locations that would be well served as Starlink users. Unlike the Toronto-Miami path, the shortest London-Johannesburg path always uses a mixture of ISLs and ground relays. The hybrid path is always better than a pure ISL path or a path that only uses ground relays.

Finally, even when ISLs always give the lowest latency path, sometimes they may not have sufficient capacity to cope with offered load. When this happens, multipath routing is needed to spread the load. If a single ISL is saturated, it may sometimes be better to use a relay to supplement the ISL rather than divert via a different satellite path.

\section{CONCLUSIONS}

Even without ISLs, LEO constellations like Starlink can provide better wide-area latency than optical fiber if ground stations can be used as relays. If idle user terminals can be used, this improves relay density and brings significant latency reduction. We proposed improvements to shortestpath routing that allow millisecond precision in path changes while scaling to sufficient relay numbers for global coverage. If ISLs are added, the need for relays is reduced, but some paths still benefit from using them, both to reduce latency, and to supplement capacity in busy parts of the network.

\section{AFTERWORD}

After this paper was accepted for publication, SpaceX again filed a request with the FCC to revise the Starlink constellation's configuration. As of October 2019, this request has not yet been approved by the FCC, but if it is approved the revised first phase will consist of 72 orbital planes, each containing 22 satellites, as opposed to 24 planes of 66 satellites analyzed in this paper. All other orbital parameters remain unchanged.

According to this latest filing, this constellation change will permit better US coverage in the early stages of deployment. Figure 14 shows this benefit - populating 18 of the 72 planes is sufficient to provide continuous US coverage, in

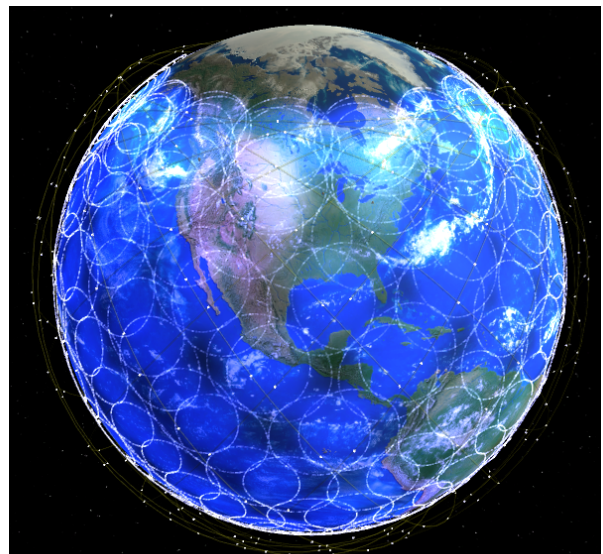

Figure 14: 22 satellites $\times 18$ planes

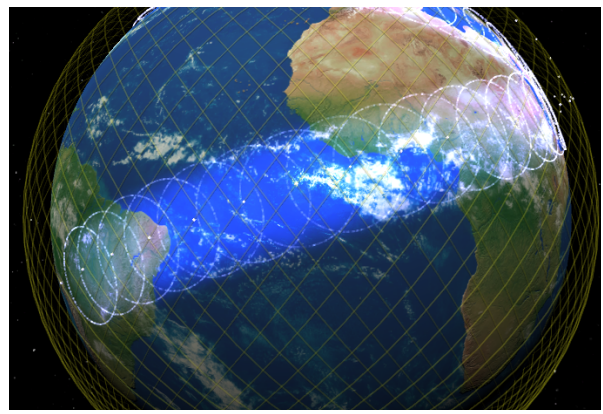

Figure 15: Overlap of the 1st satellite from each of 72 planes in August 30th 2019 FCC filing

contrast to Figure 3, which shows the same number of satellites deployed. The downside of this constellation is that coverage overlap is not so well suited for high slant-angle relays in the early stages of deployment.

As deployment progresses though, the coverage overlap needed for ground relays is regained. By using more orbital planes, the distance between neighboring planes is much reduced. Figure 15 shows all 72 orbital planes, but shows only the first satellite in each plane ${ }^{3}$. These satellites move together north-eastwards towards the northern end of their orbits, then back south-eastwards and so on, getting closer together as they move away from the equator and then further away again as they move back towards the equator. As the figure shows, at their maximum distance, the coverage overlap is very similar to that achieved using 66 satellites per plane. Thus this new constellation is also well suited to using ground stations as relays, but it prioritizes early coverage over early low-latency long distance service.

\footnotetext{
${ }^{3}$ The SpaceX FCC filing "technical parameters" database contains what appears to be an error. We simulate a 39/72 phase offset between neighboring planes, which is largely consistent with the
} database, but corrects the error. 
[1] D. Bhattacherjee, W. Aqeel, I. N. Bozkurt, A. Aguirre,

B. Chandrasekaran, P. B. Godfrey, G. Laughlin,

B. Maggs, and A. Singla. Gearing up for the $21 \mathrm{st}$ century space race. In Proceedings of the 17th ACM Workshop on Hot Topics in Networks, HotNets '18, pages 113-119, New York, NY, USA, 2018. ACM.

[2] Corning Incorporated. SMF-28TM optical fiber product information, 2002.

[3] E. W. Dijkstra. A note on two problems in connexion with graphs. Numerische Mathematik, 1:269-271, 1959.

[4] M. Handley. Delay is not an option: Low latency routing in space. In Proceedings of the 17th ACM Workshop on Hot Topics in Networks, HotNets '18, pages 85-91, New York, NY, USA, 2018. ACM.

[5] M. Handley. Starlink revisions, nov 2018. https: / / youtu . be /QEIUdMiColU, 2018.

[6] C. Henry. OneWeb's first six satellites in orbit following Soyuz launch.

https: / / spacenews.com/

first-six-oneweb-satellites-launch-on-soyuz-rocket/, 22019.

[7] T. Klenze, G. Giuliari, C. Pappas, A. Perrig, and

D. Basin. Networking in heaven as on earth. In Proceedings of the 17th ACM Workshop on Hot Topics in Networks, HotNets '18, pages 22-28, New York, NY, USA, 2018. ACM.

[8] E. Musk. Transcript of pre-launch press conference call, march 15th 2019. https: / / pastebin.com/xzGYVVRz.

[9] Space Exploration Technologies. SpaceX non-geostationary satellite system Attachment A: technical information to supplement Schedule $\mathrm{S}$.

https://licensing.fcc.gov/myibfs / download.do?attachment_key=1158350, Nov. 2016.

[10] Space Exploration Technologies. SpaceX non-geostationary satellite system Attachment A: technical information to supplement Schedule $\mathrm{S}$.

https://licensing.fcc.gov/myibfs / download.do?attachment_key=1569860, Nov. 2018.

[11] SpaceX. Starlink web site. https: / / starlink. com.

[12] WonderNetwork. Global ping statistics: Ping times between wondernetwork servers. https: / / wondernetwork. com/.

[13] H. Zech, F. Heine, D. Tröndle, S. Seel, M. Motzigemba, R. Meyer, and S. Philipp-May. LCT for EDRS: LEO to GEO optical communications at 1.8 Gbps between Alphasat and Sentinel 1a. In Proc. 